\title{
Genomic rearrangements in sporadic lymphangioleiomyomatosis: an evolving genetic story
}

Stephen J Murphy ${ }^{1}$, Simone B Terra ${ }^{1,2}$, Faye R Harris ${ }^{1}$, Aqsa Nasir ${ }^{1}$, Jesse S Voss ${ }^{2}$, James B Smadbeck ${ }^{1}$, Sarah H Johnson ${ }^{1}$, Vishnu Serla ${ }^{1}$, Jay H Ryu ${ }^{3}$, Eunhee S $\mathrm{Yi}^{2}$, Benjamin R Kipp ${ }^{2}$, George Vasmatzis ${ }^{1,4}$ and Eva M Carmona ${ }^{3,4}$

${ }^{1}$ Departments of Biomarker Discovery, Center for Individualized Medicine, Mayo Clinic, Rochester, MN, USA;

${ }^{2}$ Departments of Laboratory Medicine and Pathology, Mayo Clinic, Rochester, MN, USA and ${ }^{3}$ Departments of Pulmonary and Critical Care Medicine, Mayo Clinic, Rochester, MN, USA

Sporadic lymphangioleiomyomatosis is a progressive pulmonary cystic disease resulting from the infiltration of smooth muscle-like lymphangioleiomyomatosis cells into the lung. The migratory/metastasizing properties of the lymphangioleiomyomatosis cell together with the presence of somatic mutations, primarily in the tuberous sclerosis complex gene (TSC2), lead many to consider this a low-grade malignancy. As malignant tumors characteristically accumulate somatic structural variations, which have not been well studied in sporadic lymphangioleiomyomatosis, we utilized mate pair sequencing to define structural variations within laser capture microdissected enriched lymphangioleiomyomatosis cell populations from five sporadic lymphangioleiomyomatosis patients. Lymphangioleiomyomatosis cells were confirmed in each tissue by hematoxylin eosin stain review and by HMB-45 immunohistochemistry in four cases. A mutation panel demonstrated characteristic TSC2 driver mutations in three cases. Genomic profiles demonstrated normal diploid coverage across all chromosomes, with no aneuploidy or detectable gains/losses of whole chromosomal arms typical of neoplastic diseases. However, somatic rearrangements and smaller deletions were validated in the two cases which lacked TSC2 driver mutations. Most significantly, one of these sporadic lymphangioleiomyomatosis cases contained two different size deletions encompassing the entire TSC1 locus. The detection of a homozygous deletion of TSC1 driving a predicted case of sporadic lymphangioleiomyomatosis, consistent with the common two-hit TSC2 mutation model, has never been reported for sporadic lymphangioleiomyomatosis. However, while no evidence of the hereditary tuberous sclerosis complex disease was reported for this patient, the potential for mosaicism and sub-clinical phenotype cannot be ruled out. Nevertheless, this study demonstrates that somatic structural rearrangements are present in lymphangioleiomyomatosis disease and provides a novel method of genomic characterization of sporadic lymphangioleiomyomatosis cells, aiding in defining cases with no detected mutations by conventional methodologies. These structural rearrangements could represent additional pathogenic mechanisms in sporadic lymphangioleiomyomatosis disease, potentially affecting response to therapy and adding to the complex genetic story of this rare disease.

Modern Pathology (2017) 30, 1223-1233; doi:10.1038/modpathol.2017.52; published online 23 June 2017

Pulmonary lymphangioleiomyomatosis is a rare, systemic disease that has been classified anywhere from benign, non-neoplastic or hamartomatous, to a

Correspondence: Dr G Vasmatzis, PhD, Department of Biomarker Discovery, Center for Individualized Medicine, Mayo Clinic, Stabile 12, 200 First St SW, Rochester, MN 55905, USA or Dr EMC Porquera, MD, PhD, Pulmonary and Critical Care Medicine, Mayo Clinic, Gonda 18, 200 First St SW, Rochester, MN 55905, USA.

E-mail: vasmatzis.george@mayo.edu or carmona.eva@mayo.edu ${ }^{4}$ Co-corresponding authors contributed equally to this manuscript. Received 16 January 2017; revised 27 April 2017; accepted 27 April 2017; published online 23 June 2017 low grade, destructive and even metastasizing neoplasm. ${ }^{1-3}$ Regardless of its classification, lymphangioleiomyomatosis progressively destroys lung function of afflicted women primarily of childbearing age ${ }^{1,2}$ with an estimated median transplant-free survival of 29 years and 10-year survival of $86 \%{ }^{4}$ Lymphangioleiomyomatosis is a cystic lung disease associated with proliferation of abnormal smooth muscle cells showing coexpression of muscle and melanocytic markers. Lymphangioleiomyomatosis has been included in the wide group of proliferative lesions named perivascular epithelioid cell tumors, given the overlapping morphologic and 
immunophenotypic findings shared by lymphangioleiomyomatosis cells and perivascular epithelioid cells. Lymphangioleiomyomatosis is characterized by infiltration and proliferation of atypical smooth muscle cells (lymphangioleiomyomatosis cells) into lung structures including the lymphatics, airway walls and interstitial spaces, impairing lung function through the formation of cysts. ${ }^{1,2}$ Studies describe lymphangioleiomyomatosis as a systemic disease, with lymphangioleiomyomatosis cells originating from sources outside of the lung and potentially utilizing the lymphatic system to travel to the lung. ${ }^{1-5}$

Lymphangioleiomyomatosis occurs both sporadically or in patients with the hereditary disease tuberous sclerosis complex, and is primarily associated with pathogenic alterations in the TSC1 (hamartin) or TSC2 (tuberin) genes. ${ }^{6-8}$ These two tumor suppressors, together with $T B C 1 D 7$, form the tuberous sclerosis complex trimer that negatively regulates mechanistic target of rapamycin signaling, a pathway involved in maintaining normal cell growth/proliferation. ${ }^{9}$ Germline (hereditary) pathogenic alterations in TSC1 and TSC2 have been identified in $\sim 75-90 \%$ of individuals who meet clinical diagnostic criteria for tuberous sclerosis complex. $^{8}$ Approximately $40 \%$ of female patients with tuberous sclerosis complex also meet criteria for lymphangioleiomyomatosis on computed tomographic (CT) scans, a prevalence which was reported to increase to $80 \%$ with advancing age, with the majority of pulmonary manifestations of tuberous sclerosis complex matching those of sporadic lymphangioleiomyomatosis. ${ }^{10,11}$ The incidence of lymphangioleiomyomatosis is estimated at between 3 and 9 cases per million women with about $15 \%$ of them reportedly having tuberous sclerosis complex. ${ }^{12,13}$ The average age of diagnosis is in the early 40s with individuals with tuberous sclerosis complex lymphangioleiomyomatosis being diagnosed slightly earlier than those with sporadic Lymphangioleiomyomatosis. ${ }^{12,13}$ Individuals with tuberous sclerosis complex lymphangioleiomyomatosis also tend to develop symptoms at a slightly earlier age. ${ }^{13}$ Tuberous sclerosis complex lymphangioleiomyomatosis primarily encompasses germline alterations in TSC2 and less frequently in TSC1. ${ }^{6-8,10,11,14-16}$ Sporadic lymphangioleiomyomatosis, however, is primarily considered a TSC2 disease. ${ }^{6-8}$

Individuals with sporadic lymphangioleiomyomatosis (that is, no clinical diagnosis of tuberous sclerosis complex and no germline alterations in TSC1/2) frequently harbor two somatic TSC2 alterations in their lymphangioleiomyomatosis tissue, consistent with the two-hit tumor suppressor model. ${ }^{17}$ Biallelic inactivation of either TSC1 or TSC2 is also frequent in tuberous sclerosis complex lymphangioleiomyomatosis, with the first a tuberous sclerosis driving germline mutation and the second a somatic tuberous sclerosis complex lymphangioleiomyomatosis driving mutation. ${ }^{18}$ The distinction between sporadic and tuberous sclerosis complex lymphangioleiomyomatosis is fundamentally the diagnosis of tuberous sclerosis complex disease, however, this diagnosis is sometimes complex. Firstly, TSC1 mutation driven tuberous sclerosis frequently results in a milder form of disease than TSC2 mutation driven tuberous sclerosis. ${ }^{19}$ Secondly, somatic and germline mosaicism for TSC1/2 mutations have been described in a sub-population of tuberous sclerosis complex patients and their parents, respectively. ${ }^{20}$

Although the majority of lymphangioleiomyomatosis tissues are characteristically TSC1 or TSC2 mutated, a significant number of cases (10-15\%) still present with no mutations in these genes suggesting undetected mutagenic events. ${ }^{20}$ While large deletions and duplication in TSC1 and TSC2 have been reported in tuberous sclerosis complex, ${ }^{21}$ the involvement of somatic large genomic rearrangements have not been well studied in lymphangioleiomyomatosis disease. Large genomic rearrangements frequently encompass key driver events of tumor initiation and progression in tumors, ${ }^{2-25}$ including noninvasive adenocarcinoma in situ lesions of the lung. ${ }^{25}$ Thus, we initially hypothesized that large genomic rearrangements can occur somatically in the lymphangioleiomyomatosis cell genome and could play a role in disease pathogenesis through their impact on genes in the mechanistic target of rapamycin or related pathways. We also hypothesized that these somatic rearrangements could have been missed in previous lymphangioleiomyomatosis cell genomic studies based on point mutations and/or local copy-number changes in the tuberous sclerosis complex genes and thus could define somatic variations in cases where no mutations were previously detected. Through the partnering of laser capture microdissection and whole-genome amplification techniques we have developed robust protocols to enable genome-wide profiling of structural variation in defined cell populations with limited cell numbers. ${ }^{23,25-27}$ Through the application of whole-genome mate pair sequencing, we investigated the genomic landscape of enriched populations of lymphangioleiomyomatosis cells and adjacent control histologically normal smooth muscle cells from the lung tissues of five sporadic lymphangioleiomyomatosis patients.

\section{Materials and methods}

\section{Tissue Immunohistochemistry}

Fresh frozen lung tissues from five sporadic lymphangioleiomyomatosis cases were obtained from the national disease research interchange tissue bank. No tuberous sclerosis complex was detailed for any of the patients in the national disease research interchange tissue bank clinical notes (Supplementary Table 1). No national disease 
research interchange tissue bank tissue codes for tuberous sclerosis complex were listed for any patient upon tissue banking. In the absence of available formalin fixed tissue from the five cases, five-micron sections were cut from these fresh frozen lung tissue samples and stained with hematoxylin eosin stain to evaluate the morphologic features and verify the diagnosis of lymphangioleiomyomatosis. Plump spindle-shaped myoid cells with pale eosinophilic cytoplasm characterize the morphologic features of lymphangioleiomyomatosis cells. As the majority of lymphangioleiomyomatosis cells are positive for the melanocytic marker HMB- $45^{28}$ each case also underwent a standard immunohistochemical staining with the HMB-45 anti-human monoclonal mouse antibody (Dako, Carpinteria, CA, USA; 1:100 dilution) using 3,3'-diaminobenzidine as chromogen and hematoxylin as counterstaining.

\section{DNA Isolation and Sequencing}

Guided by the hematoxylin eosin and HMB-45 staining, laser capture microdissection was used to independently isolate enriched populations of lymphangioleiomyomatosis cells and control histologically normal smooth muscle cells from sequential 10-micron fresh frozen tissue sections of each patient. Laser capture microdissection of the control histologically normal smooth muscle cells was performed on the same laser capture microdissection slide as the lymphangioleiomyomatosis cells, but in areas where no abnormal lymphangioleiomyomatosis cell pathology was observed. Limitations and the potential for lymphangioleiomyomatosis cell contamination in this control are presented in discussion. DNA was amplified directly from the captured cells by a single-step procedure using a modified Qiagen Repli-g protocol and $1 \mathrm{\mu g}$ used to assemble indexed Illumina Nextera Mate Pair libraries, as previously described. ${ }^{23-27}$ Libraries were sequenced two per lane on the HiSeq $2000(2 \times 101 \mathrm{bp})$. Paired reads were mapped to the $\mathrm{Hg} 38$ reference genome as previously described. ${ }^{21,29}$ Discordant mate pairs mapping $>15 \mathrm{~kb}$ apart or in different chromosomes were selected for further analysis. Genomic breakpoint junctions detected with 7 or greater mate pair read associates were considered to have a high confidence and low probability of being false positives. A mask was used to eliminate common variants and discordant mate pairs from experimental or algorithmic errors. ${ }^{29}$ Primers spanning detected fusion junctions were used in PCR validations on lymphangioleiomyomatosis, smooth muscle cells and benign epithelial tissue (when available) and an independent human Genomic DNA control (G304A; Promega, Madison, WI, USA). TSC1/2 mutation panel: DNA from LAM tissues for each case was analyzed for mutations in the TSC1 and TSC2 genes using a gene panel. DNA from the control smooth muscle cell tissues for each case was also tested to support the clinically reported absence of tuberous sclerosis disease in these patients. Nextgeneration sequencing libraries were prepared using $50 \mathrm{ng}$ of whole-genome amplified DNA and a GeneRead DNAseq Custom Panel V2 kit (Qiagen) encompassing the coding regions of 50 cancer-associated genes: AKT1, ATRX, BRAF, CDKN2A, CDKN2B, CIC, CTNNB1, DAXX, EGFR, FGFR1, FGFR2, FGFR3, FUBP1, GNA11, GNAQ, GNAS, H3F3A, IDH1, IDH2, JAK2, MYBL1, MYC, MYCN, NF1, NF2, NOTCH1, NOTCH2, PDGFRA, PIK3CA, PIK3R1, PIK3R2, PTCH1, PTEN, RB1, SDHA, SDHB, SDHC, SDHD, SMARCA4, SMARCB1, SMO, STAT3, SUFU, TERT, TET1, TET2, TP53, TSC1, TSC2 and WT1. PCR products were further processed using the TruSeq Nano DNA Library Preparation kit, final libraries were pooled equimolar and underwent $2 \times 101$ bp sequencing on HiSeq 2500. Raw sequencing data was processed by a CLC Bio Genomics Server using custom-built bioinformatics pipelines. Variants with $\geq 10 \%$ mutant allele frequency were reviewed to determine pathogenicity status following the current standards and guidelines for the interpretation of sequence variants. ${ }^{30}$

\section{Results}

\section{Histological Assessment of Lymphangioleiomyomatosis} Tissues

Abnormal smooth muscle cells highly indicative of lymphangioleiomyomatosis cells were observed in each case by pathology review of hematoxylin eosin stained tissues (Figure 1; Table 1). HMB-45 immunostaining was positive in these abnormal smooth muscle cells in all but one case (LAM2). There was strong HMB-45 staining in LAM3, LAM4 and LAM5, with weaker staining in LAM1. A representative hematoxylin eosin stain from the fresh frozen tissue is presented in Figure 1f. Sufficient numbers of lymphangioleiomyomatosis cells and control histologically normal smooth muscle cells were successfully isolated from each tissue to yield genomic DNA through the direct whole-genome amplified methodology.

\section{TSC1 and TSC2 Mutation Profiling}

While no TSC1 mutations were observed, somatic mutations in the TSC2 gene were observed in three lymphangioleiomyomatosis tissues, LAM1, LAM2 and LAM5 (Table 1), which were not detected in the germline control normal smooth muscle cells. LAM1 and LAM2 each contained two damaging mutations in the TSC2 gene, supporting the two-hit tumor suppressor model. A pathogenic TSC2 splice variant mutation with additional frame shift and premature stop mutations were identified in LAM1 and LAM2, respectively. A single somatic frame shifting pathogenic mutation was observed in TSC2 of LAM5. Just one further pathogenic mutation in the 

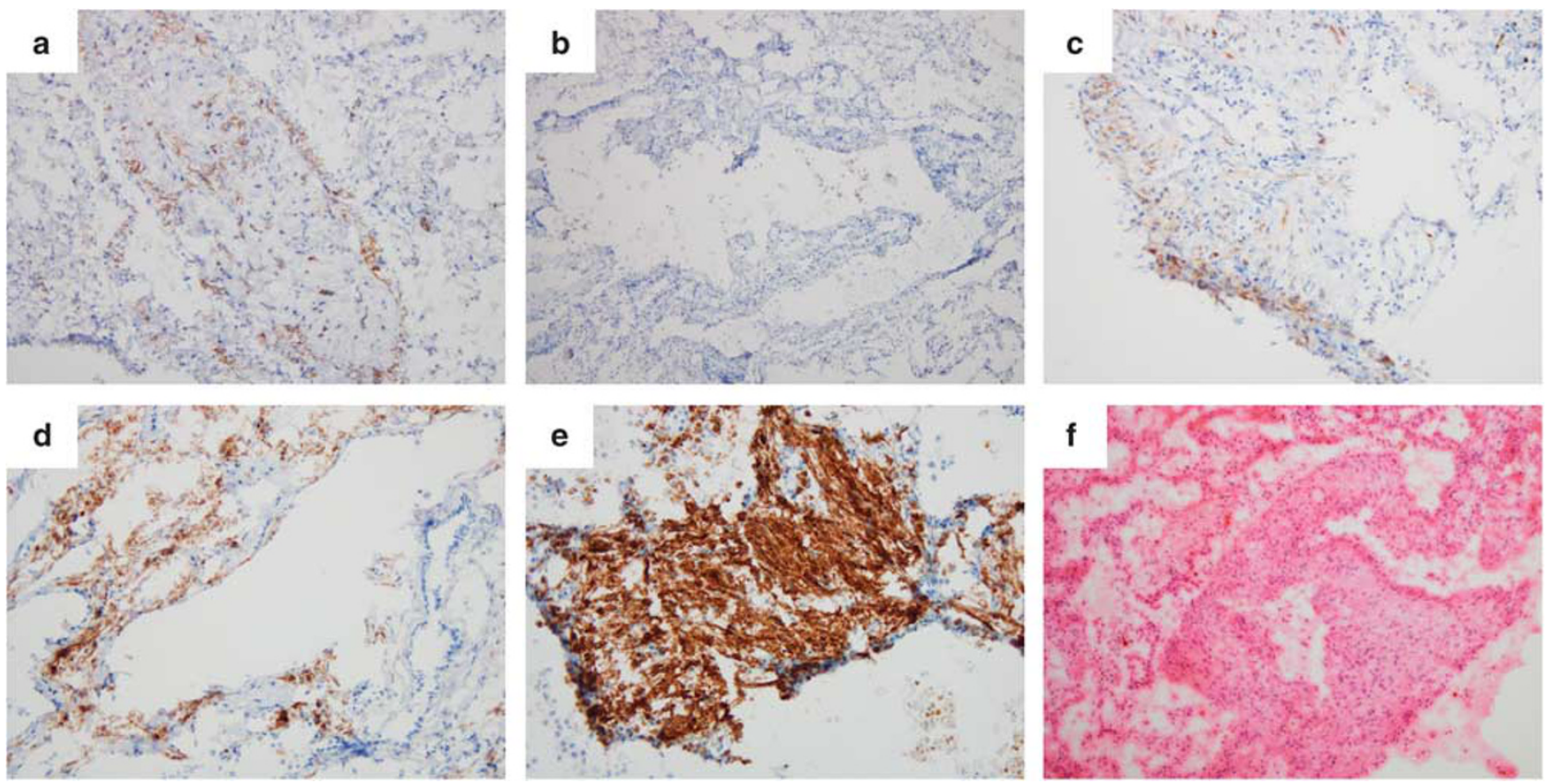

Figure 1 Histological review of lymphangioleiomyomatosis tissues. Immunohistochemical staining images of lymphangioleiomyomatosis tissues from cases LAM1-LAM5 (a-e) with the HMB-45 antibody. HMB-45 staining was absent from LAM2 (b). Representative hematoxylin eosin staining of LAM5 from fresh frozen tissue (f).

additional genes in the panel was observed in TET2 of LAM5, which was not present in the associated smooth muscle cells (Table 1). No germline pathogenic variations were detected in the TSC1 or TSC2 genes. Thus, both the clinical data and genomic panel analysis supports the reported absence of tuberous sclerosis disease in these patients.

The variant frequency for the observed somatic mutations in LAM1, LAM2 and LAM5 predicted lymphangioleiomyomatosis cell purity in each sample in the range of $35 \%, 30 \%$ and $20 \%$, respectively (Table 1). Visual inspection of the sequencing raw read data for the remaining two cases (LAM3 and LAM4) yielded no evidence of additional low frequency TSC1 or TSC2 mutations.

\section{Genomic Landscape of Lymphangioleiomyomatosis Tissues}

In order to assess the involvement of large genomic rearrangements in lymphangioleiomyomatosis disease, DNA from each lymphangioleiomyomatosis tissue and three selected smooth muscle cell tissues were used for structural variance analysis. The Illumina mate pair sequencing protocol was used to elucidate genome-wide structural variations within the tumors. Mate pair sequencing spans the genome with larger spanning genomic fragments than conventional genomic sequencing, increasing the potential of detecting discordantly mapping breakpoints from rearrangements. ${ }^{29}$ Mate pair sequencing data generated an average of 90 million reads per sample, with high mapping efficiency to the human reference genome (Supplementary Table 2). An average bridged coverage across the genomes of $80 \times$ was observed, enabling high confidence profiling of structural variations from the reference human genome.

Genome plots present the landscape of structural variation within the lymphangioleiomyomatosis cell, displaying coverage frequency across each chromosome $^{29}$ (Figure 2a-e). No significant evidence of aneuploidy was observed in any of the lymphangioleiomyomatosis samples. Small regions of gains and loss were evident, but the majorities of these copy variations involved normal natural variation from the reference genome and were shared in associated smooth muscle cell samples (Supplementary Figure 1). Figure $2 \mathrm{f}$ exemplifies a typical genome plot of a metastatic lung adenocarcinoma, to demonstrate the extensive aneuploidy, focal gains, losses and chromosomal translocations frequently observed in neoplastic tissues.

Structural genomic rearrangements result in discordant mapping fusion junctions absent from the reference genome, which can be efficiently mapped from mate pair sequencing data. Each lymphangioleiomyomatosis tissue contained very few structural variations, with genome profiles very similar to the normal smooth muscle cells. In LAM1, LAM2 and LAM5, with detected TSC2 mutations, no somatic fusion junctions were determined (Table 2). Just three events passed bioinformatics filters in LAM1 and LAM2, which were confirmed germline through shared presence in the associated smooth muscle cells (Table 2). Five events passed filters for LAM5, which in the absence of mate pair sequencing data 
Table 1 Summary of histological review of LAM tissues and results of a gene sequencing panel for TSC1 and TSC2 mutations

\begin{tabular}{|c|c|c|c|c|c|c|c|}
\hline \multirow{2}{*}{$\begin{array}{l}\text { LAM } \\
\text { case }\end{array}$} & \multirow{2}{*}{$\begin{array}{l}L A M \text { cells } \\
H \& E \\
\text { staining }\end{array}$} & \multirow{2}{*}{$\begin{array}{l}\text { HMB- } 45 \\
\text { stain }\end{array}$} & \multirow{2}{*}{$\begin{array}{l}\text { Pathogenic } \\
\text { TSC1/2 } \\
\text { germline } \\
\text { mutations }\end{array}$} & \multirow{2}{*}{$\begin{array}{l}\text { TSC1 } \\
\text { somatic } \\
\text { mutations }\end{array}$} & \multicolumn{2}{|l|}{ TSC2 somatic mutations } & \multirow{2}{*}{$\begin{array}{l}\text { Other panel } \\
\text { somatic mutations }\end{array}$} \\
\hline & & & & & Mutation site & $\begin{array}{l}\text { Variant } \\
\text { frequency }\end{array}$ & \\
\hline 1 & Present & $\begin{array}{l}\text { Weakly } \\
\text { Positive }\end{array}$ & None & None & TSC2 c.4569G > T, Splice Variant & 0.25 & None \\
\hline 2 & Present & Negative & None & None & $\begin{array}{l}\text { TSC2 c.5084dup, p.Asp1696Gly } \\
\text { TSC2 c.3094C > T, p.Arg1032X } \\
\text { TSC2 c.3610G > A, Splice Variant }\end{array}$ & $\begin{array}{l}0.11 \\
0.16 \\
0.14\end{array}$ & None \\
\hline 3 & Present & Positive & None & None & None & NA & None \\
\hline 4 & Present & Positive & None & None & None & NA & None \\
\hline 5 & Present & Positive & None & None & TSC2 c.1835del p.Leu612ArgfsX86 & 0.11 & $\begin{array}{l}\text { TET2 c.3789T }>A, p . \\
\text { Cys1263X (Variant } \\
\text { frequency } 0.10 \text { ) }\end{array}$ \\
\hline
\end{tabular}

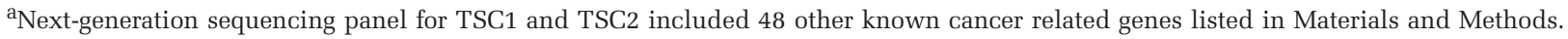

on the associated smooth muscle cells, were not automatically determined as germline variations. Further PCR validation of three selected events confirmed them as germline through their presence in the associated SMC5 DNA (Figure 3a). One of these events involved $P P P 2 R 2 B$, a gene implicated in mechanistic target of rapamycin signaling. ${ }^{31,32}$

In LAM3 and LAM4, where no somatic or germline tuberous sclerosis complex gene mutations were detected, somatic rearrangements were detected by the mate pair sequencing and selected events successfully validated by PCR (Table 2; Figure 3a-d). A total of eight events passed bioinformatics filters for LAM4, with just one confirmed germline event being present in SMC4. For the seven somatic events, no reads were detected in the associated smooth muscle cells, and selected events were confirmed somatic by PCR validation (Figure 3a). Most significantly, two events predicted nested deletions across the TSC1 gene locus, indicative of a double loss (Figure 3b, c and e). Copy-number variation coverage confirmed a definite loss of heterozygosity at the TSC1 locus not present in the smooth muscle cell tissue (Figure 3f). One of these deletions additionally predicted an $A B O-D D X 31$ gene fusion. Other genes directly hit included; GFI1B, AK8 and SERPINA11 (Table 2).

Somatic fusion junctions were also verified in LAM3 (Figure 3a). Three fusion junctions were initially predicted to be somatic (Table 2) including a PCR validated event on chromosome 5 (Figure 3d) and two events located on the X chromosome. While PCR validation of the $\mathrm{X}$ chromosome events were impeded by the repetitive nature of the DNA region involved, copy-number variation did concur with the rearrangement site (Figure 3g). The overlapping rearrangements predicted both deletion and gain within the $0.8 \mathrm{Mb}$ region of Xp11.3 (Figure 3g). An additional translocation event between chromosomes 1 and 5, which did not initially pass filters due to just four mate pair reads spanning the junction, was also PCR validated as somatic in LAM3 (Figure 3a).

No evidence of loss of heterozygosity was apparent at the TSC2 locus on chromosome $16 \mathrm{p} 13.3$ or $T B C 1 D 7$ at 6 p24.1 in any case. Loss of heterozygosity at the TSC1 locus on chromosome 9q34.13 was only evident in LAM4, supporting the deletions reported. No additional loss of heterozygosity was observed at other major genes implicated in the mechanistic target of rapamycin signaling pathway (data not shown).

\section{Discussion}

We report here the first in-depth genome-wide study of structural variation within sporadic lymphangioleiomyomatosis cell genomes. While loss of heterozygosity at the TSC1/2 loci have been reported in select studies, ${ }^{7,14}$ the overall involvement of large genomic rearrangements in lymphangioleiomyomatosis disease has not been well studied. Previous studies on genomic structural variations in lymphangioleiomyomatosis cells have focused primarily on the tuberous sclerosis complex gene loci and primerbased amplicon sequencing techniques. ${ }^{7,14}$ Specifically, Kozlowski and colleagues utilized multiplex ligation-dependent probe amplification, which required multiple probe sets specific to each TSC1/2 exon. The mate pair sequencing technique employed in this study enabled a simpler genome-wide evaluation of structural variation within laser capture microdissection enriched populations on lymphangioleiomyomatosis cells and comparison with normal smooth muscle cells from the same patient. Significantly, somatic rearrangements were observed as active in sporadic lymphangioleiomyomatosis disease, being present in two cases lacking any evidence of TSC2 driver mutations. Selected events were validated by PCR and confirmed as absent from adjacent normal smooth muscle cells from the same 
a

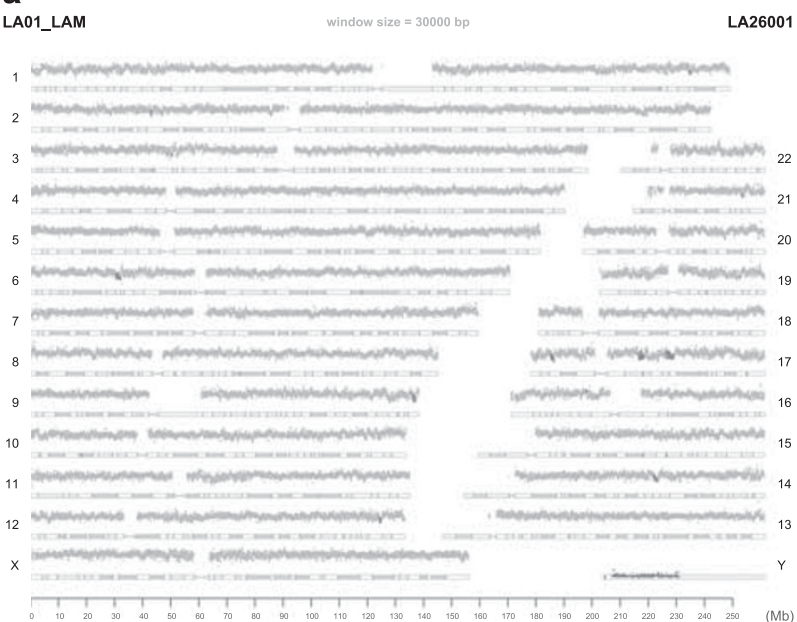

C

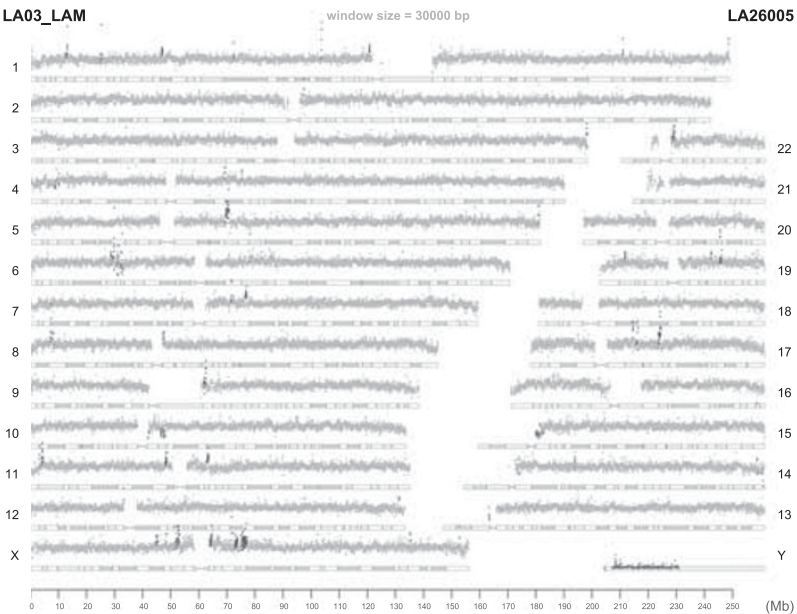

e

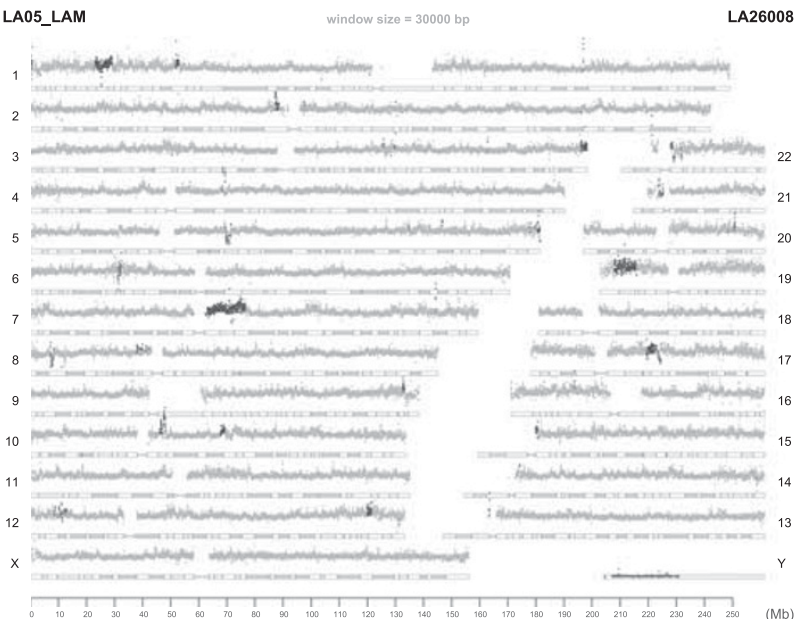

b

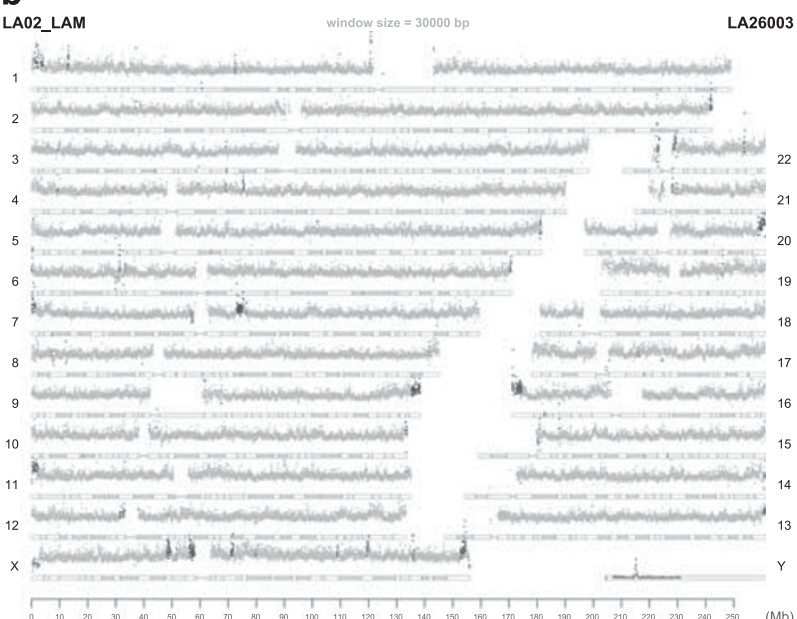

\section{d}

LA04 LAM

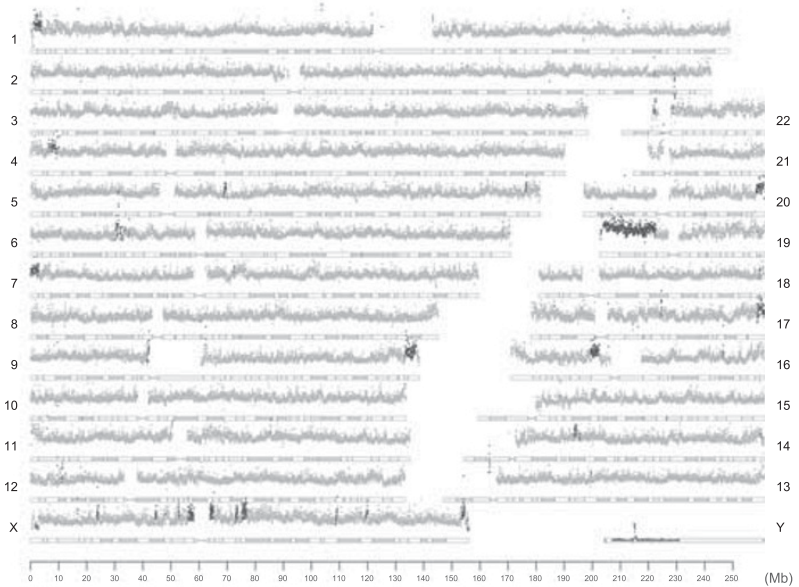

f

LU135_ADSQ_a $\quad$ window size $=30000$ bp $\quad$ LU20301

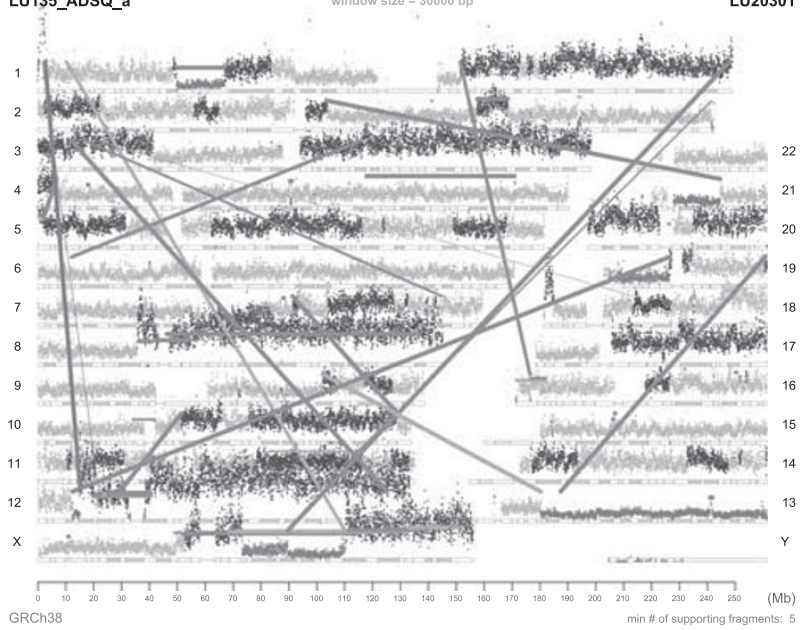

Figure 2 Genome plots of lymphangioleiomyomatosis cells. Genome plots presenting coverage across the 22 chromosomes and the $\mathrm{X}$-chromosomes of the lymphangioleiomyomatosis cells from LAM1-LAM5 (a-e) and a representative lung adenocarcinoma (AD) (f). Coverage frequency (y-axis) for $30 \mathrm{~kb}$ windows across each chromosome ( $x$-axis) are presented horizontally. The central gray coverage indicates normal heterozygous two copy of a chromosome. Gains and losses are observed as darker regions of raised or dropped coverage. Inter-chromosomal large genomic rearrangement translocations are presented as black lines linking different chromosomes. Intra-chromosomal rearrangements are presented as black lines above the specific chromosomal positions involved. A full color version of this figure is available in the online version of this paper. 
Table 2 Genomic rearrangements in S-LAM tissues

\begin{tabular}{|c|c|c|c|c|c|c|c|}
\hline \multirow{2}{*}{ Case } & \multicolumn{2}{|c|}{$\# M P$ reads } & \multirow{2}{*}{$\begin{array}{l}\text { Rearrangement junctions locus } 1 \\
\text { (Position 1)-locus 2(Position 2) }\end{array}$} & \multirow{2}{*}{$\begin{array}{c}\text { Span } \\
(K b)\end{array}$} & \multirow{2}{*}{ Genes in region } & \multirow{2}{*}{ Description } & \multirow{2}{*}{ Event } \\
\hline & $L A M$ & $S M C$ & & & & & \\
\hline \multirow[t]{2}{*}{1} & 17 & 8 & 12p11.21 (31850695)-12p11.21 (31909225) & 59 & Non-genic & Inversion gain & Germline \\
\hline & 25 & 26 & 13q21.1 (57568991)-13q21.1 (57619680) & 51 & Non-genic & Inversion gain & Germline \\
\hline 2 & 47 & 48 & 5q21.1 (101791596)-5q21.1 (101851281) & 60 & Non-genic & Inversion gain & Germline \\
\hline \multirow[t]{6}{*}{3} & 10 & ND & $7 \mathrm{p} 22.2(3873982)-7 \mathrm{p} 22.2(3936243)$ & 62 & SDK1 & Inversion gain & Germline \\
\hline & 7 & ND & 5q21.3 (109342051)-11q12.1 (59911834) & NA & PJA2 & Transposon & Germline \\
\hline & 63 & ND & Xp11.3 (44425634)-Xp11.3 (44909659) & 484 & $\begin{array}{l}\text { KDM6A, FUNDC1, DUSP21, } \\
\text { CXorf36 }\end{array}$ & Complex deletion & Somatic $^{\mathrm{a}}$ \\
\hline & 22 & ND & Xp11.3 (44435731)-Xp11.3 (45205625) & 770 & & Complex deletion & Somatic $^{\mathrm{a}}$ \\
\hline & 4 & ND & $1 q 41(222152591)-5 q 32(148341148)$ & NA & LOC102546294 & Translocation & Somatic ${ }^{b}$ \\
\hline & 13 & ND & 5q31.1 (132242829)-5q31.1 (132277952) & 35 & PDLIM4 & Inversion gain & Somatic $^{\mathrm{b}}$ \\
\hline \multirow[t]{8}{*}{4} & 10 & 11 & Xp22.33 (841881)-Xp22.33 (1204564) & 363 & CRLF2 & Inversion & Germline \\
\hline & 11 & 0 & 8p22 (15910687)-8p22 (15990423) & 80 & Non-genic & Inversion & Somatic \\
\hline & 16 & 0 & 9q31.1 (100671056)-9q31.1 (100820933) & 150 & Non-genic & Inversion & Somatic \\
\hline & 9 & 0 & 9q31.1 (100698800)-9q31.1 (100829569) & 131 & Non-genic & Inversion & Somatic \\
\hline & 9 & 0 & 9q34.13 (132728341)-9q34.13 (132977378) & 249 & AK8/GFI1B & Deletion & Somatic $^{\mathrm{b}}$ \\
\hline & 15 & 0 & $9 q 34.13$ (132624957)-9q34.2 (133257990) & 633 & DDX31/ABO & Deletion & Somatic \\
\hline & 25 & 0 & $14 q 32.13$ (94443781)-14q32.2 (98082349) & 3639 & SERPINA11 & Inversion & Somatic ${ }^{\mathrm{b}}$ \\
\hline & 16 & 0 & 14q32.13 (94455998)-14q32.2 (98088865) & 3633 & Non-genic & Inversion & Somatic \\
\hline \multirow[t]{5}{*}{5} & 88 & ND & $18 q 22.1(68177018)-18 q 22.1(68230580)$ & 54 & Non-genic & Deletion & Germline \\
\hline & 23 & ND & $4 q 28.3(136293092)-12 p 13.32$ (3614684) & NA & CRACR2A & Transposon & Germline \\
\hline & 45 & ND & $5 q 32(146549343)-5 q 32(146699199)$ & 150 & PPP2R2B & Inversion gain & Germline $^{b}$ \\
\hline & 31 & ND & $\begin{array}{c}12 \mathrm{q} 24.33(131243084)-12 \mathrm{q} 24.33 \\
(131345405)\end{array}$ & 102 & LOC338797 & Deletion & Germline $^{b}$ \\
\hline & 15 & ND & Xq12 (67413801)-Xq12 (67516929) & 103 & Non-genic & Inversion gain & Germline ${ }^{b}$ \\
\hline
\end{tabular}

Abbreviations: LAM, LCM captured lymphangioleiomyomatosis cells; ND, not determined; NA, not applicable; SMC, LCM captured normal smooth muscle cells.

aPCR validation prevented by repetitive region.

bselected PCR validated event.

patients. As the control, histologically normal smooth muscle cells were collected from adjacent tissue to the lymphangioleiomyomatosis histology for each case, we cannot rule out the possibility of contamination of small numbers of lymphangioleiomyomatosis cells in these controls. However, for cases LAM1, LAM2 and LAM5 where TSC2 point mutations were detected in the lymphangioleiomyomatosis tissues, no supporting reads were detected in the smooth muscle cell controls. Similarly, for LAM4, no supporting mate pair reads were detected for the two TSC1 deletion events in SMC4. While LAM3 did not contain a somatic TSC1 or TSC2 event to assess contamination of lymphangioleiomyomatosis cells in the SMC3 control, rearrangement events present in LAM3 failed to validate by PCR in SMC3. Thus, these observations provided confidence that laser capture microdissection of histologically normal smooth muscle cells from the same tissue sections where the lymphangioleiomyomatosis cells were isolated did not significantly contaminate with lymphangioleiomyomatosis cells.

Biallelic inactivation of either TSC1 or TSC2 has been well established as the principal drivers of lymphangioleiomyomatosis disease; however, the complex nature of this disease still presents many unanswered questions. Although the majority of lymphangioleiomyomatosis show genomics supporting the two-hit tumor suppressor inactivation model, a subset of lymphangioleiomyomatosis still lack identifiable mutations. Although more recent deep sequencing studies in previously described no mutation identified cases identified low-prevalence somatic exonic mutations ${ }^{18}$ or non-coding intronic splice effector mutations ${ }^{20}$ in tuberous sclerosis complex genes, cases are still reported in these studies with no defined driver events. Additionally, while sporadic lymphangioleiomyomatosis disease is characterized as solely a TSC2 mutation driven disease, with no reported involvement of TSC1 mutations, diagnosis of sporadic lymphangioleiomyomatosis in absence of tuberous sclerosis complex is complicated by potential mosaicism and lower clinical presentation of TSC1-driven disease in a small subset of tuberous sclerosis complex cases. ${ }^{19,20}$ A small sub-population of patients with this rare sporadic lymphangioleiomyomatosis disease is thus subsequently diagnosed with sub-clinical tuberous sclerosis complex lymphangioleiomyomatosis.

The presence of lymphangioleiomyomatosis cells in each case was confirmed by histological review of hematoxylin eosin and HMB-45 stained tissue sections by experienced pathologists. Both available clinical data and genomic analysis on control smooth muscle cell tissue from each patient inferred an absence of tuberous sclerosis complex in all of these patients, supporting the diagnosis of sporadic lymphangioleiomyomatosis. The presence of somatic 
a

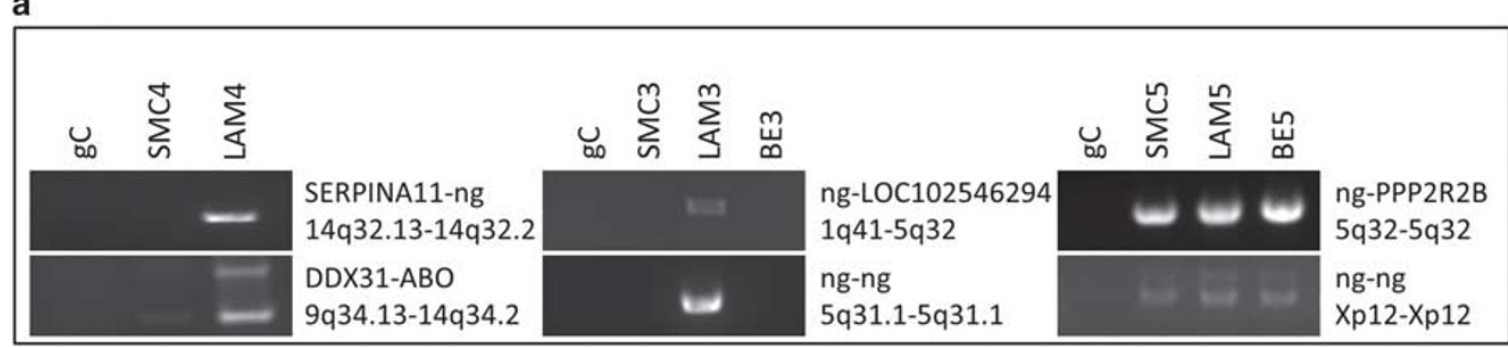

b

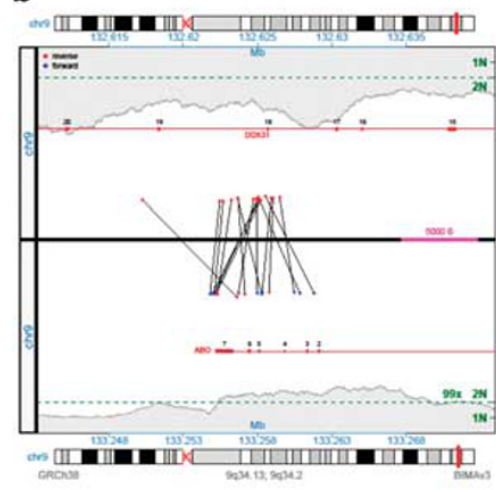

e

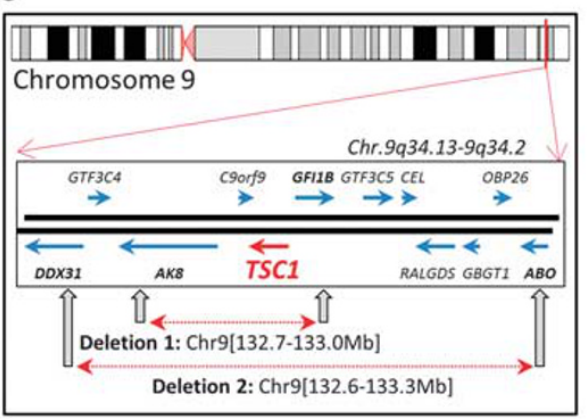

c
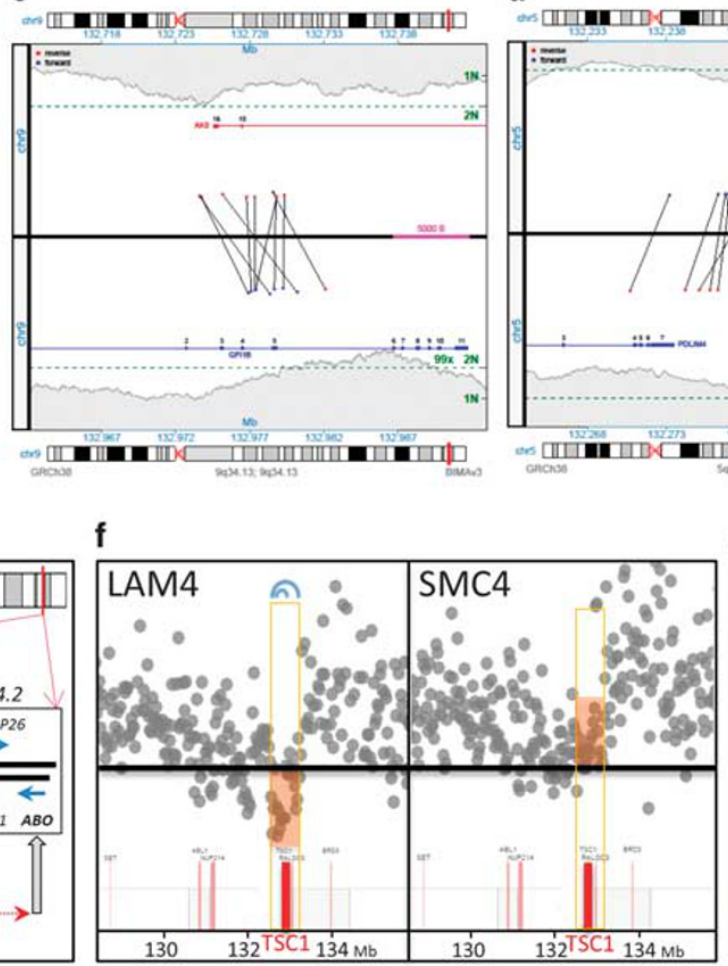

d

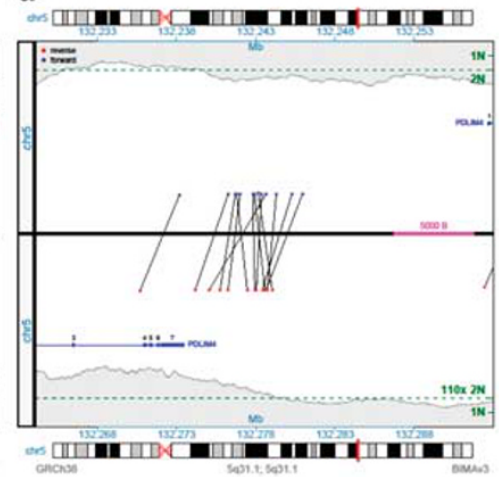

g

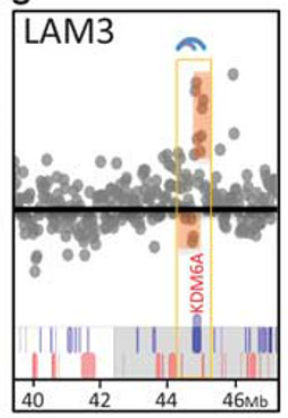

Figure 3 Somatic rearrangements in lymphangioleiomyomatosis tissues. (a) PCR validations using primers spanning breakpoint junctions determined in lymphangioleiomyomatosis, smooth muscle cells or benign epithelium from LAM3, LAM4 and LAM5. The genes and chromosomal loci are presented beside each gel image (ng; no gene). (b-d) Junction plots of the two deletions spanning TSC1 in LAM4 and rearrangement on chromosome 5 of LAM3. The upper and lower panels of the junction plots present the two breakpoint locations on chromosome 9 or 5 with the associated mate pair reads mapping at each region imaged as red (forward strand mapping) and blue (reverse strand mapping) dots in the upper and lower panels, respectively, linked by black lines. Genes located in each breakpoint region are indicated with exon numbers. (e) Schematic of the two TSC1 spanning deletions in LAM4 showing the position of each breakpoint and the genes involved. (f,g) Focused local coverage at the chromosome 9 TSC1 locus for LAM4 and SMC4 and the KDM6A chromosome X locus of LAM3. The small blue arched images in the upper region for the LAM4 and LAM3 samples present the span of the two detected rearrangements in each sample, which are outlined by the orange rectangles with orange shaded areas demonstrating the comparative loss of heterozygosity.

point mutations in LAM1, LAM2 and LAM5 from the mutational panel, made evaluation of the proportion of lymphangioleiomyomatosis cells captured possible. Even after enrichment by laser capture microdissection, the proportion of lymphangioleiomyomatosis cells still ranged from $20-35 \%$ in LAM1, LAM2 and LAM5, emphasizing the limitation of genomic studies on lymphangioleiomyomatosis tissues in the absence of enrichment protocols. The absence of somatic mutations from the panel in LAM3 and LAM4, however, made lymphangioleiomyomatosis cellularity predictions more subjective. Strong positive HMB-45 staining in both LAM3 and LAM4, however, confirmed the presence of lymphangioleiomyomatosis cells in these two cases. Additionally, germline events in mate pair sequencing data often easily distinguish through their characteristic features, which allow us to predict the nature of events that passed filters (Table 2). While the mutation panel failed to detect any evidence of tuberous sclerosis complex gene mutations in LAM3 or LAM4 the validation of somatic structural variations in these tissues supports the presence of adequate levels of lymphangioleiomyomatosis cells for mutational 
analysis. The supporting loss of heterozygosity at the TSC1 locus of LAM4 and Xp11.3 locus of LAM3 (Figure 3) also support the presence of adequate lymphangioleiomyomatosis cells for evaluation of copy-number variation and aneuploidy. Aneuploidy, involving gains and losses of whole chromosomes or large regions of chromosomes is characteristic of systemic tumors, ${ }^{24}$ but was not evident of any of the cases in this study. While whole-genome amplified instills some noise in the copy-number variation analysis, bioinformatics algorithms were designed to minimize this noise and effectively assess copy gains and losses. ${ }^{33}$

The observed double hits on the TSC2 gene in LAM1 and LAM2 were consistent with the two-hit tumor suppressor driver model. ${ }^{17}$ The homozygous deletion of TSC1 in LAM4 was also consistent with this model and confirms a role of large genomic rearrangements in lymphangioleiomyomatosis disease. LAM5, however, with just a single point mutation detected on TSC2, could infer an undetected mutation on the second allele of TSC2. With the mechanistic target of rapamycin signaling pathway as the major driver of the proliferative lymphangioleiomyomatosis disease, many patients benefit from sirolimus or more recently everolimus treatment; however, the drugs are sometimes poorly tolerated and aimed at cytostatic rather than cytoablative therapy. ${ }^{14,15,34}$ No alternative effective clinical protocols currently exist for lymphangioleiomyomatosis, with advanced patients often benefitting from lung transplantation. ${ }^{4,16}$ There is therefore a need to identify specific molecular targets to offer patients additional therapeutic strategies. While additional genes were hit through the large genomic rearrangements detected (Table 2) the roles as drivers in sporadic lymphangioleiomyomatosis are not wellsupported by this study, as such statements would require more in-depth supporting mechanistic studies. However, it is clear that additional genes are mutated in sporadic lymphangioleiomyomatosis disease adding large genomic rearrangements to the complex genetic story of drivers of the lymphangioleiomyomatosis phenotype. One of these events involved PPP2R2B, implicated in mechanistic target of rapamycin signaling and reported as a biomarker for rapamycin responsiveness. ${ }^{31,32}$ Other novel genes hit by rearrangements with links to mechanistic target of rapamycin signaling included DDX31, GFI1B, KDM6A, TET2, CRLF2 and CRAC2RA $A^{31,32,35-41}$ While driver roles are not predicted for these genes, it could be hypothesized that such mutations could affect disease presentation and/or potential response to treatment therapies. The additional validation of a fusion junction with just four associated mate pair reads in LAM3 also indicates a potential lower concentration of LAM cells or heterogeneity within this specimen. In samples with low cellularity or with fusion junctions in repetitive/complex regions of the genome, true positive events can appear with lower numbers of supporting associate mate pair reads.
Generally with mate pair sequencing data conservative filtering is applied to minimize false positives, however, no significant mechanistic target of rapamycin signaling pathway genes were elucidated even in the lower level associate events (data not shown). Therefore, while somatic tuberous sclerosis complex gene driver mutations were discernible in four of the five cases, LAM3 fell into the category of undetermined driver.

We believe mate pair sequencing will be instrumental in aiding detection of somatic variations in a subset of lymphangioleiomyomatosis cases where no TSC1 or TSC2 mutations were detected by standard genomic testing. These cases are the most interesting in defining additional pathways/mechanisms which drive lymphangioleiomyomatosis disease. In our study LAM3 and LAM4 fell under this category, and mate pair was able to efficiently detect the driver TSC1 mutations in LAM4. The driver in LAM3, however, remained undetermined; this case would greatly benefit from further in-depth integrated genomic analysis, including whole exome and transcriptome sequencing, to further dissect somatic variations in this and similar undefined cases. While care must be taken in over interpreting novel mutated genes in this small study, commonality in expanded case studies would aid in distinction between passenger and driver events.

In this study, we potentially report for the first time a sporadic lymphangioleiomyomatosis case driven by a homozygous TSC1 deletion. This event was predicted somatic by PCR, being absent in the germline control smooth muscle cell tissue, with coverage data also corroborating the copy loss. However, we acknowledge that care must be taken in this conclusion. Firstly, the requirement of fresh frozen tissue necessitated us to utilize lung tissue obtained from the NDRI tissue bank, which negated us access to full clinical records or additional patient tissues. While we can confirm the homozygous TSC1 deletion absent from the surrounding normal tissue, we cannot rule out the potential for mosaicism. Secondly, although consultation with NDRI strongly indicated no tuberous sclerosis complex disease in any of the patients studied, as detailed in Supplementary Table 2, the fact that TSC1 mutation driven tuberous sclerosis complex can present with sub-clinical tuberous sclerosis complex, additionally prevents us from categorically ruling out mosaicism in this patient. Furthermore, the fact that TSC1 mutations are less common in tuberous sclerosis complex than TSC2-driven, and that TSC1-dirven mutations could potentially present with subclinical sporadic lymphangioleiomyomatosis disease, together with the rarity of this disease, may also explain the lack of reporting of a TSC1-driven sporadic lymphangioleiomyomatosis to date.

In summary, we report here an analysis of structural variation in sporadic lymphangioleiomyomatosis cells. Results definitively demonstrated the involvement of large genomic rearrangements/ 
deletions in a subset of sporadic lymphangioleiomyomatosis cases lacking the well reported TSC2 driver mutations. Most significantly, we observed a homozygous deletion of TSC1 in a wild type TSC2 background, potentially demonstrating for the first time a case of sporadic lymphangioleiomyomatosis driven by two-hits on the TSC1 gene. More importantly, these somatic mutations would have been missed with most technologies used to screen sporadic lymphangioleiomyomatosis tissues. The numbers of somatic rearrangements was, however, limited and not present in all cases. Nevertheless, somatic genomic rearrangements are active in sporadic lymphangioleiomyomatosis and should not be overlooked in the genomic analysis, specifically in no mutation identified cases, for which mate pair sequencing is a useful tool to evaluate all structural variations in one single sequencing procedure.

\section{Acknowledgments}

Work was supported by John Adler (Adler Foundation) and Mayo Clinic Centre for Individualized Medicine. We would also like to thank Bruce Eckloff and Bob Sikkink from the Mayo Genomics Sequencing Core for the mate pair sequencing.

\section{Disclosure/conflict of interest}

The authors declare no conflict of interest.

\section{References}

1 Ferrans VJ, Yu ZX, Nelson WK, et al. Lymphangioleiomyomatosis (LAM): a review of clinical and morphological features. J Nippon Med Sch 2000;67:311-329.

2 Moir LM. Lymphangioleiomyomatosis: current understanding and potential treatments. Pharmacol Ther 2016;158:114-124.

3 McCormack FX, Travis WD, Colby TV, et al. Lymphangioleiomyomatosis: calling it what it is: a low-grade, destructive, metastasizing neoplasm. Am J Respir Crit Care Med 2012;186:1210-1212.

4 Oprescu N, McCormack FX, Byrnes S, et al. Clinical predictors of mortality and cause of death in lymphangioleiomyomatosis: a population-based registry. Lung 2013;191:35-42.

5 Karbowniczek M, Astrinidis A, Balsara BR, et al. Recurrent lymphangiomyomatosis after transplantation: genetic analyses reveal a metastatic mechanism. Am J Respir Crit Care Med 2003;167:976-982.

6 Strizheva GD, Carsillo T, Kruger WD, et al. The spectrum of mutations in TSC1 and TSC2 in women with tuberous sclerosis and lymphangiomyomatosis. Am J Respir Crit Care Med 2001;163:253-258.

7 Badri KR, Gao L, Hyjek E, et al. Exonic mutations of TSC2/TSC1 are common but not seen in all sporadic pulmonary lymphangioleiomyomatosis. Am J Respir Crit Care Med 2013;187:663-665.

8 Sato T, Seyama K, Fujii H, et al. Mutation analysis of the TSC1 and TSC2 genes in Japanese patients with pulmonary lymphangioleiomyomatosis. J Hum Genet 2002;47:20-28.

9 Dibble CC, Elis W, Menon S, et al. TBC1D7 is a third subunit of the TSC1-TSC2 complex upstream of mTORC1. Mol Cell 2012;47:535-546.

10 Costello LC, Hartman TE, Ryu JH. High frequency of pulmonary lymphangioleiomyomatosis in women with tuberous sclerosis complex. Mayo Clin Proc 2000;75: 591-594.

11 Cudzilo CJ, Szczesniak RD, Brody AS, et al. Lymphangioleiomyomatosis screening in women with tuberous sclerosis. Chest 2013;144:578-585.

12 Ryu JH, Moss J, Beck GJ, et al. The NHLBI lymphangioleiomyomatosis registry: characteristics of 230 patients at enrollment. Am J Respir Crit Care Med 2006;173:105-111.

13 Johnson SR, Taveira-DaSilva AM, Moss J. Lymphangioleiomyomatosis. Clin Chest Med 2016;37:389-403.

14 Carsillo T, Astrinidis A, Henske EP. Mutations in the tuberous sclerosis complex gene TSC2 are a cause of sporadic pulmonary lymphangioleiomyomatosis. Proc Natl Acad Sci USA 2000;97:6085-6090.

15 Yates DH. mTOR treatment in lymphangioleiomyomatosis: the role of everolimus. Expert Rev Respir Med 2016;10:249-260.

16 Ussavarungsi $\mathrm{K}$, $\mathrm{Hu} \mathrm{X}$, Scott $\mathrm{P}$, et al. Mayo clinic experience of lung transplantation in pulmonary lymphangioleiomyomatosis. Respir Med 2015;109:1354-1359.

17 Knudson AG. Two genetic hits (more or less) to cancer. Nat Rev Cancer 2001;1:157-162.

18 Fujita A, Ando K, Kobayashi E, et al. Detection of lowprevalence somatic TSC2 mutations in sporadic pulmonary lymphangioleiomyomatosis tissues by deep sequencing. Hum Genet 2016;135:61-68.

19 Muzykewicz DA, Sharma A, Muse V, et al. TSC1 and TSC2 mutations in patients with lymphangioleiomyomatosis and tuberous sclerosis complex. J Med Genet 2009;46:465-468.

20 Tyburczy ME, Dies KA, Glass J, et al. Mosaic and intronic mutations in TSC1/TSC2 explain the majority of TSC patients with no mutation identified by conventional testing. PLoS Genet 2015;11:e1005637.

21 Kozlowski P, Roberts P, Dabora S, et al. Identification of 54 large deletions/duplications in TSC1 and TSC2 using MLPA, and genotype-phenotype correlations. Hum Genet 2007;121:389-400.

22 Vasmatzis G, Johnson SH, Knudson RA, et al. Genome-wide analysis reveals recurrent structural abnormalities of TP63 and other p53-related genes in peripheral T-cell lymphomas. Blood 2012;120: 2280-2289.

23 Murphy SJ, Aubry MC, Harris FR, et al. Identification of independent primary tumors and intrapulmonary metastases using DNA rearrangements in non-smallcell lung cancer. J Clin Oncol 2014;32:4050-4058.

24 Murphy SJ, Hart SN, Halling GC, et al. Integrated genomic analysis of pancreatic ductal adenocarcinomas reveals genomic rearrangement events as significant drivers of disease. Cancer Res 2016;76:749-761.

25 Murphy SJ, Wigle DA, Lima JF, et al. Genomic rearrangements define lineage relationships between adjacent lepidic and invasive components in lung adenocarcinoma. Cancer Res 2014;74:3157-3167.

26 Murphy SJ, Cheville JC, Zarei S, et al. Mate pair sequencing of whole-genome-amplified DNA following laser capture microdissection of prostate cancer. DNA Res 2012;19:395-406. 
27 Murphy SJ, Hart SN, Lima JF, et al. Genetic alterations associated with progression from pancreatic intraepithelial neoplasia to invasive pancreatic tumor. Gastroenterology 2013;145:1098-109 e1.

28 Tanaka H, Imada A, Morikawa $\mathrm{T}$, et al. Diagnosis of pulmonary lymphangioleiomyomatosis by HMB45 in surgically treated spontaneous pneumothorax. Eur Respir J 1995;8:1879-1882.

29 Drucker TM, Johnson SH, Murphy SJ, et al. BIMA V3: an aligner customized for mate pair library sequencing. Bioinformatics 2014;30:1627-1629.

30 Richards S, Aziz N, Bale S, et al. Standards and guidelines for the interpretation of sequence variants: a joint consensus recommendation of the American College of Medical Genetics and Genomics and the Association for Molecular Pathology. Genet Med 2015;17:405-424.

31 Hosseini SM, Dufort I, Caballero J, et al. Transcriptome profiling of bovine inner cell mass and trophectoderm derived from in vivo generated blastocysts. BMC. Dev Biol 2015;15:49.

32 Qian XJ, Li YT, Yu Y, et al. Inhibition of DNA methyltransferase as a novel therapeutic strategy to overcome acquired resistance to dual PI3K/mTOR inhibitors. Oncotarget 2015;6:5134-5146.

33 Smadbeck JB, Johnson SH, Smoley SA, et al. Copy number variant analysis using genome-wide mate-pair sequencing. (in preparation)

34 McCormack FX, Gupta N, Finlay GR, et al. Official American Thoracic Society/Japanese Respiratory Society
Clinical Practice Guidelines: Lymphangioleiomyomatosis Diagnosis and Management. Am J Respir Crit Care Med 2016;194:748-761.

35 Tasian SK, Doral MY, Borowitz MJ, et al. Aberrant STAT5 and PI3K/mTOR pathway signaling occurs in human CRLF2-rearranged B-precursor acute lymphoblastic leukemia. Blood 2012;120:833-842.

36 Luik RM, Wang B, Prakriya M, et al. Oligomerization of STIM1 couples ER calcium depletion to CRAC channel activation. Nature 2008;454:538-542.

37 Zhang Y, Tu Y, Zhao J, et al. Reversion-induced LIM interaction with Src reveals a novel Src inactivation cycle. J Cell Biol 2009;184:785-792.

38 Takayama K, Misawa A, Suzuki T, et al. TET2 repression by androgen hormone regulates global hydroxymethylation status and prostate cancer progression. Nat Commun 2015;6:8219.

39 Fukawa T, Ono M, Matsuo T, et al. DDX31 regulates the p53-HDM2 pathway and rRNA gene transcription through its interaction with NPM1 in renal cell carcinomas. Cancer Res 2012;72:5867-5877.

40 Khandanpour C, Phelan JD, Vassen L, et al. Growth factor independence 1 antagonizes a p53-induced DNA damage response pathway in lymphoblastic leukemia. Cancer Cell 2013;23:200-214.

41 Martin D, Abba MC, Molinolo AA, et al. The head and neck cancer cell oncogenome: a platform for the development of precision molecular therapies. Oncotarget 2014;5:8906-8923.

Supplementary Information accompanies the paper on Modern Pathology website (http://www.nature.com/ modpathol) 